\title{
An automated algorithm for extracting road edges from terrestrial mobile LiDAR data
}

\author{
Pankaj Kumar*, Conor P. McElhinney, Paul Lewis, Timothy McCarthy \\ National Centre for Geocomputation (NCG), National University of Ireland Maynooth (NUIM), Maynooth, Co. Kildare, Ireland
}

\section{A R T I C L E I N F O}

\section{Article history:}

Received 25 March 2013

Received in revised form 13 August 2013

Accepted 13 August 2013

Available online 8 September 2013

\section{Keywords:}

Edge

Automation

Extraction

LiDAR

Terrestrial mobile

\begin{abstract}
A B S T R A C T
Terrestrial mobile laser scanning systems provide rapid and cost effective 3D point cloud data which can be used for extracting features such as the road edge along a route corridor. This information can assist road authorities in carrying out safety risk assessment studies along road networks. The knowledge of the road edge is also a prerequisite for the automatic estimation of most other road features. In this paper, we present an algorithm which has been developed for extracting left and right road edges from terrestrial mobile LiDAR data. The algorithm is based on a novel combination of two modified versions of the parametric active contour or snake model. The parameters involved in the algorithm are selected empirically and are fixed for all the road sections. We have developed a novel way of initialising the snake model based on the navigation information obtained from the mobile mapping vehicle. We tested our algorithm on different types of road sections representing rural, urban and national primary road sections. The successful extraction of road edges from these multiple road section environments validates our algorithm. These findings and knowledge provide valuable insights as well as a prototype road edge extraction toolset, for both national road authorities and survey companies.

(c) 2013 International Society for Photogrammetry and Remote Sensing, Inc. (ISPRS) Published by Elsevier
\end{abstract} B.V. All rights reserved.

\section{Introduction}

Light Detection And Ranging (LiDAR) is a relatively recent technology, enabling 3D surveying of real world environments by measuring the time of return of emitted light pulses. Laser scanning systems make use of this technology to acquire accurately georeferenced sets of dense 3D LiDAR point cloud data. They provide several benefits over conventional sources of data acquisition in terms of accuracy, resolution, attributes and automation. The information obtained through laser scanning systems have applications ranging from monitoring urban development to evaluating natural environments (Darnel, 2012).

Laser scanning systems are used to acquire LiDAR data from aerial and terrestrial platforms. The data acquired from these systems differs in terms of its intrinsic accuracy and resolution for a variety of reasons but primarily due to the distance of the scanner to the target objects (Rutzinger et al., 2009). In recent years, the use of laser scanners onboard terrestrial based moving vehicles has led to an increase in the collection of high quality 3D data. The applicability of these terrestrial Mobile Mapping Systems (MMSs) continue to prove their worth in route corridor, urban and utility

\footnotetext{
* Corresponding author. Tel.: +353 17086731.

E-mail address: pankaj.kumar@nuim.ie (P. Kumar).
}

mapping due to the rapid, continuous and cost effective 3D data acquisition capability compared with static terrestrial laser scanning systems (Haala et al., 2008; Barber et al., 2008).

The concept of terrestrial MMS dates back to 1990s and since then has been primarily driven by the advances in kinematic positioning, imaging, laser scanning, data fusion and spatial information technologies. Mobile mapping refers to a means of collecting geospatial data using mapping and navigation sensors that are mounted rigidly onboard a mobile platform (Tao and Li, 2007). Mapping sensors can consist of imaging and laser scanning system while the navigation system is based on integrated Global Navigation Satellite System (GNSS) and Inertial Navigation System (INS). In their initial development phase, terrestrial MMSs were developed based on GNSS/INS integration and digital cameras. Later, several MMSs integrated with laser scanners were reported over subsequent years. The development of various terrestrial MMSs has been thoroughly reviewed in Ellum and El-Sheimy (2002) and Barber et al. (2008). Over the last 20 years, terrestrial MMSs have slowly developed from research projects in the academic sector to becoming commercially viable activities (Petrie, 2010). There are a number of companies which provide rapid and cost effective capturing of 3D LiDAR data for larger areas. According to a market research study conducted by ARC advisory group, the 3D laser scanning market is expected to double in size from 2010 to 2015 (Rio, 2011). 
Terrestrial MMSs present a reliable and rapid tool for capturing 3D spatially referenced information about road infrastructure and surrounding route corridor environment. This information can assist road authorities in effective management of road networks and to ensure maximum safe driving condition for road users. The volume of data produced by a terrestrial mobile laser scanning system such as the Riegl VQ-250 is large, generating 300,000 points per second resulting in approximately $20 \mathrm{~GB}$ of data per hour. Manual processing of such data is time consuming and has led to the development of automated algorithms. To estimate road geometry and physical road objects along the route corridor, road edges are needed to be correctly identified and extracted. The road edge is a fundamental feature and its efficient extraction is a topic which has not been extensively explored by the research community. In this paper we present an algorithm which has been developed for extracting road edges in multiple road environments from terrestrial mobile LiDAR data. The algorithm is based on the novel combination of two modified versions of the parametric active contour or snake model that, in turn, provides a more precise extraction of the road edges. In Section 2, we review various methods developed for extracting the road and its boundaries from LiDAR data. In Section 3, we discuss the parametric active contour models and investigate associated methods that have been developed for segmentation from LiDAR data. Following the review, we list the research limitations in current approaches which are addressed in our research work. In Section 4, we provide a step wise description of our road edge extraction algorithm. In Section 5 , we describe the process for validating the extracted road edges. We test our road edge extraction algorithm on different types of road sections in Section 6. In Section 7, we validate and discuss the road edge extraction test results. Finally, we draw a number of conclusions in Section 8.

\section{Previous work on road extraction}

The use of LiDAR technology for mapping route corridors provides accurate and dense $3 \mathrm{D}$ point cloud data which can be used for reliable and precise extraction of road features. The methods developed for segmenting LiDAR data are mostly based on the identification of planar or smooth surfaces and the classification of point cloud data based on its attributes (Vosselman, 2009). Based on these methods, several attempts have been made to extract the road and associated features from LiDAR data. Clode et al. (2004) segmented airborne LiDAR point cloud data into road and non-road objects using a hierarchical classification technique based on elevation and intensity information. The accuracy of their road segmentation approach was reduced due to the presence of car parks and private roads in their survey area. Goulette et al. (2006) presented a method for segmenting road, trees and facades from terrestrial mobile LiDAR data. The road was segmented as horizontal plane with high density of points in the histogram and then the segmented information was used to compute road width and curvature. The trees and facades were identified as vertical planes and disconnected elements in the histogram. Yuan et al. (2008) proposed an algorithm for extracting road surface from terrestrial LiDAR data. The algorithm used a fuzzy clustering method to cluster LiDAR points. Straight lines were then fitted to the linearly clustered data using slope information for extracting the road surface area. Elberink and Vosselman (2009) developed an automated method for 3D modelling of highway infrastructure using airborne LiDAR and 2D topographic map data. The road polygons were extracted from the topographic map data using a map based seed-growing algorithm combined with a Hough transformation. The LiDAR points were added to the corresponding road polygons using a LiDAR based seed-growing algorithm. Subsequently, 3D reconstruction was achieved by assigning the LiDAR elevation values to the map polygons. Lam et al. (2010) extracted roads from terrestrial mobile LiDAR data by fitting RANdom SAmple Consensus (RANSAC) planes to small sections and then interconnected these fitted planes using Kalman filtering. The extracted road information was further used to segment urban objects such as lamp posts, power line posts and power lines based on dimensional constraint and fitting RANSAC lines to 3D points.

Few research attempts have been focused on precisely extracting the road edges. Jaakkola et al. (2008) developed a method for classifying kerbstones, road surface model and road markings from terrestrial mobile LiDAR data. The kerbstones were delineated by filtering the gradient image of height attribute to find the pixels which were neither horizontal nor vertical. The extracted kerbstone information was used to estimate the points that belong to the road surface area. These points were used to create a triangulated irregular network and then it was shaped into smooth surface by applying slope and edge length constraints. The road markings were extracted by first normalising the intensity attribute and then applying threshold approach. Yoon et al. (2009) presented an approach for evaluating the terrain surface for autonomous vehicles in an urban environment from LiDAR point cloud data. They calculated the slope and standard deviation from LiDAR points and used these values to estimate the edges of the road. Vosselman and Liang (2009) developed a method for detecting kerbstones from airborne LiDAR data. The approach was based on the detection of small height jumps caused by the kerbstones in the LiDAR point cloud data. However, their extraction accuracy was affected by parked cars occluding the kerbstones. Smadja et al. (2010) developed an algorithm for extracting roads from LiDAR data based on the detection of slope break points coupled with the RANSAC algorithm. The extracted road boundaries were further processed to compute road curvature and road width information. Zhang (2010) proposed a method for detecting road edges in an urban environment using terrestrial LiDAR data. In their method, road edge points were identified based on elevation information. The identified 3D road edge points were then projected on a ground plane to estimate the road kerbs. McElhinney et al. (2010) developed an algorithm for extracting road edges from terrestrial mobile LiDAR data. In the first stage of their algorithm, a set of lines were fitted to the road cross sections based on the navigation data and then LiDAR points within the vicinity of the lines were determined. In the second stage, these points were analysed based on information such as the slope, intensity, pulse width and proximity to vehicle information in order to extract the road edges. Ibrahim and Lichti (2012) developed an approach to extract street kerb and its surface from terrestrial mobile LiDAR point cloud data. In their approach, the point cloud data was segmented into ground and non-ground based on varying point density with respect to the distance from mobile vehicle's trajectory. The segmented ground data was further refined by analysing the morphological characteristics of the neighbourhood of each point in the segment. The kerb edges were finally extracted from the refined ground segment by applying the derivative of the Gaussian function to 3D points.

The majority of these road extraction methods attempt to delineate roads by distinguishing them from non-road objects but do not make attempt to extract the road edges. Approaches which have been developed for extracting road edges to date fail to provide an efficient and robust solution. Most of these approaches have been developed for urban road environments where algorithms rely on the existence of a sufficient height or slope difference between the road and kerb points for detecting road edges. Little or no research has been carried out to extract rural roads, where the non-road surface comprises grass-soil and the edges are not as easily defined by slope changes alone. The intensity and pulse width attributes from LiDAR data can be a useful source 
of information for extracting these road edges. Their use in urban and rural road sections has yet to be thoroughly explored. In the next section, we describe parametric active contour models and review approaches based on them that has been developed for extracting features from LiDAR data.

\section{Parametric active contour models}

Parametric active contour or snake models are robust segmentation approach which make efficient use of specific information available about objects in the input data rather than processing all the data (Blake and Isard, 1998). Their concept was first introduced by Kass et al. (1988) in which the snake is represented explicitly as a controlled spline curve and is implemented based on computed energy. The snake curve is defined within a 2D image domain that moves towards a desired object boundary under the influence of an internal energy within the curve itself and an external energy derived from the image data.

The snake is defined parametrically in the $x, y$ plane of an image as

$v(s)=[x(s), y(s)]$,

where $v(s)$ represents the snake curve, $s$ is its normalised arc length and $x(s), y(s)$ are coordinates along the snake curve. The curve $v(s)$ is represented by a set of control points $v_{0}, v_{1}, \ldots, v_{m-1}$ and is linearly obtained by joining each control point (Kumar, 2012). The snake's energy function, $E_{\text {snake, }}$, constituting the internal and external energy terms is described as

$E_{\text {snake }}=\int_{0}^{m-1}\left(E_{\text {int }}(v(s))+E_{\text {ext }}(v(s))\right) d s$.

The internal energy, $E_{i n t}$ controls the snake curve's elasticity and stiffness properties which can be written as

$E_{\text {int }}=\frac{1}{2}\left(\alpha(s)\left|\frac{d v}{d s}\right|^{2}+\beta(s)\left|\frac{d^{2} v}{d s^{2}}\right|^{2}\right)$,

where $\alpha(s)$ and $\beta(s)$ are weight parameters. The $\alpha$ weight parameter controls elasticity while the $\beta$ weight parameter controls stiffness in the snake curve. The external energy, $E_{\text {ext }}$, is estimated from the gradient image of object boundaries that attracts the snake curve toward it. The image gradient based external energy is described as

$E_{\text {ext }}=-\kappa|\nabla f(v(s))|^{2}$,

where $\kappa$ is a weight parameter and $\nabla f$ is the gradient image of object boundaries, $f$. The snake's energy function is discretised and solved iteratively (Xu and Prince, 1998). The $\gamma$ weight parameter is used to control the snake curve step size in one iteration which can be written as

$\gamma=\frac{\mu \cdot \delta t}{\delta x \cdot \delta y}$

where $\mu$ is a regularisation parameter which controls the impact of any noise present in the image, $\delta t$ is a time step for each iteration and $\delta x, \delta y$ represents space between image points ( $\mathrm{Xu}$ and Prince, 1998). An increased noise in the image will require a higher value for $\mu$. The energy of the snake is minimised by maintaining a state balance between the internal and external energy terms. When the snake's energy function reaches a minimum, it converges to the object boundary (Kumar et al., 2010).

The traditional parametric active contour model is associated with two limitations in object boundary estimation (Xu and Prince, 1998). First, the snake curve must be initialised close to a desirable object boundary or else it won't converge to the object boundary. Second, the snake curve fails to detect concave boundaries. Several method have been proposed to overcome these limitations. Cohen (1991) proposed a balloon concept in the parametric active contour model in which an additional energy is added to the external energy term which pushes a snake curve towards an object boundary. The balloon energy acts in the normal direction to a point on the curve which makes the behaviour of the snake curve more dynamic. The balloon energy can be described as

$E_{\text {balloon }}=\kappa_{1} \cdot n(s)$

where $\kappa_{1}$ is the weight parameter for the balloon energy and $n(s)$ is the unit vector normal to the snake curve at point $v(s)$. The balloon model overcomes the snake curve initialisation drawback but does not provide a solution for the concave boundary convergence problem.

Xu et al. (1997) introduced a Gradient Vector Flow (GVF) external energy in the parametric active contour model in an attempt to detect the concave boundaries. It is computed as a diffusion of the gradient vectors of the object boundaries which attracts the snake curve toward the object boundaries (Kumar et al., 2010). The GVF energy is described as

$E_{G V F}=\iint \mu\left(u_{x}^{2}+u_{y}^{2}+w_{x}^{2}+w_{y}^{2}\right)+|\nabla f|^{2}|V-\nabla f|^{2} d x d y$,

where $V(x, y)=(u(x, y), w(x, y))$ is the energy field and $u, w$ are its vector components in the $x, y$ plane of an image. When $\nabla f$ is small or negligible, the energy function is dominated by the first term which is a sum of the squares of partial derivatives of the vector components. When $\nabla f$ is large, the second term dominates and minimises the energy function, when $V=\nabla f$. It results in a smoothly varying energy being produced over a homogeneous image region while not affecting the gradient energy along the object boundary. The diffused energy allows the snake curve to detect the concave boundaries and helps increase the movement range of the snake curve. The GVF model can overcome both the limitations in the traditional parametric active contour model, however, its ability to overcome the snake initialisation problem is limited in the case where there is a relatively large distance between the initial snake and the object boundaries.

Several methods based on snake curves have been developed for extracting road and building objects from LiDAR data. Goepfert and Rottensteiner (2009) applied a snake model to extract the road network for integrating and matching, digital landscape model derived from $2 \mathrm{D}$ vector data and digital terrain model generated from airborne LiDAR data. In their approach, the snake curve was initialised near the road feature using existing vector data, stored as a polyline, while the external energy terms were derived from intensity, elevation and surface roughness based on images generated from the LiDAR data. Kabolizade et al. (2010) proposed an improved snake model for extracting buildings from aerial images and LiDAR data. The initial estimation of building edges was carried out by applying a threshold to the normalised digital surface model. The snake model was implemented by deriving the external energy terms based on intensity and altitude variances of the snake curve points with their neighbourhood points. Boyko and Funkhouser (2011) described a method for extracting roads from a large scale 3D point cloud data merged from aerial and terrestrial laser scanning of an urban environment. An initial approximation of the road network in the point cloud data was made using 2D map and then the road network was divided into independent patches to make the process computationally efficient. In each patch, an active contour model was applied by estimating the elevation based attractor function as an external energy of the snake curve to find the kerbs in the urban road sections.

Snake curves present a useful approach for extracting objects from LiDAR data. The development of an automated method for initialising the snake curves will overcome the requirement of 
manual intervention or a priori information for their initialisation. There is a need to develop a more robust approach for determining object boundaries based image gradient values which are used to estimate external energy term. LiDAR data provides elevation, intensity and pulse width information. Further investigation of these attributes is required in order to derive the external energy terms. In the next section, we describe our road edge extraction algorithm which attempts to address the limitations in the current approaches.

\section{Algorithm}

Our algorithm is based on the novel combination of GVF and balloon parametric active contour models, to extract road edges from terrestrial mobile LiDAR data. We use the LiDAR elevation, reflectance and pulse width attributes in the algorithm which are converted into $2 \mathrm{D}$ raster surfaces to reduce computational expense. For example, one of a $30 \times 10 \times 5 \mathrm{~m}$ section of LiDAR data consists of 92,737 points, thus an iterative estimation of the GVF external energy for each point attribute would be computationally expensive. We use the reflectance attribute which is a representation of the normalised intensity values with respect to standard reflector targets (Rieger et al., 2010). We input $n$ number of $30 \times 10 \times 5 \mathrm{~m}$ LiDAR and $n$ number of $10 \mathrm{~m}$ navigation data sections in the algorithm. The dimensions of input data sections were selected based on empirical tests as they impact the process efficiency in terms of computational cost. The selection of $30 \mathrm{~m}$ width ensured the inclusion of road in the data while $5 \mathrm{~m}$ elevation was useful in order to remove the impact of vertical objects such as tress, road signs, light poles along the route corridor on the raster surface interpolation. In order to find the optimal length, we analysed three test cases in which a temporal performance of our algorithm was assessed in a road section with $10 \mathrm{~m}, 20 \mathrm{~m}$ and $30 \mathrm{~m}$ length (Kumar, 2012). In the $10 \mathrm{~m}, 20 \mathrm{~m}$ and $30 \mathrm{~m}$ cases, the snake curve required around 623,1570 and $3191 \mathrm{~s}$ respectively to converge to the road edges. This analysis was performed on a computer with two Intel Xeon E5607 processors @2.27 GHz, 12 GB RAM and a 64-bit operating system. For example, to process a $60 \mathrm{~m}$ section of road with $30 \times 10 \times 5 \mathrm{~m}$ would require approximately $3738 \mathrm{~s}$, with $30 \times 20 \times 5 \mathrm{~m}$ would require $4710 \mathrm{~s}$ and with $30 \times 30 \times 5 \mathrm{~m}$ would require $6382 \mathrm{~s}$, without overlap. Thus, on the basis of computational cost, we chose $10 \mathrm{~m}$ as an optimal length of the road section to which our road edge extraction algorithm can be applied efficiently. The input data sections were selected with an overlap of $2 \mathrm{~m}$ between them which allows us to batch process consecutive and overlapped road sections as required in the algorithm. A work flow of the road edge extraction algorithm is shown in Fig. 1. In the following sections, we describe the various processing steps involved in our algorithm.

\subsection{Terrain pyramids generation}

LiDAR data can be affected by high frequency noise due to a lower vertical accuracy of points relative to their horizontal sampling distance (Crawford, 2009). In terrestrial mobile laser scanning system, the scanner position, operating speed, configuration and scanner distance can also effect the horizontal sampling distance. Our mobile mapping research group at National University of Ireland Maynooth (NUIM) is involved in the process of finding the effect, that these factors have on the acquired point density

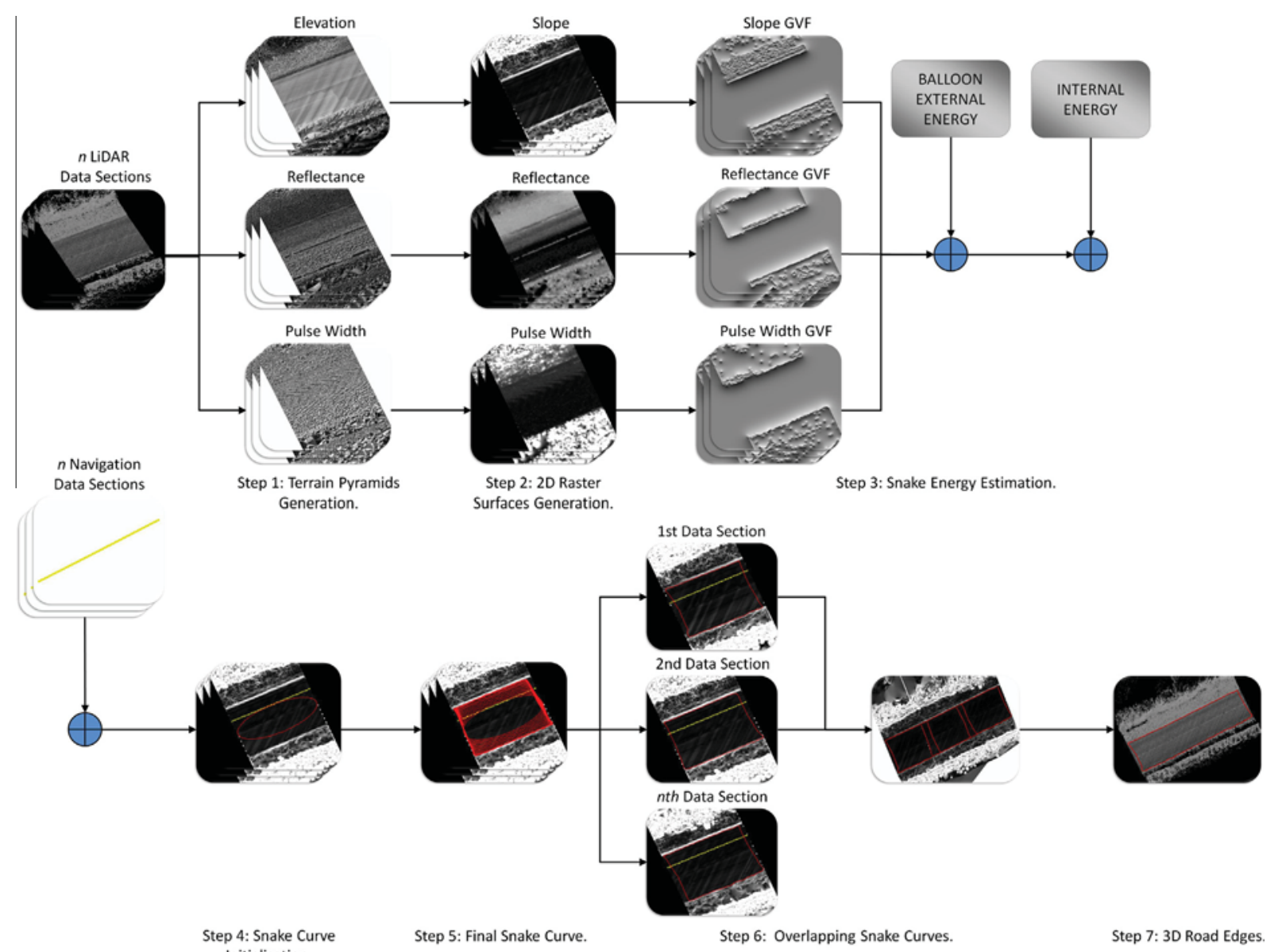


(Cahalane et al., 2010; Cahalane et al., 2011). LiDAR data can also suffer from unusual intensity and pulse width values. This high frequency noise present in LiDAR data can lead to the generation of noisy raster surfaces. We minimise the effect of this noise through a point thinning process (Crawford, 2009). Point thinning generates multi resolution terrain pyramid by reducing the number of LiDAR points required to represent a terrain model in each pyramid level (ESRI, 2010). In Step 1 of our algorithm, we generate multi resolution terrain pyramids from the LiDAR attributes where the original full resolution corresponds to the highest resolution terrain model, the first level corresponds to the second-highest resolution terrain model and the last level corresponds to the lowest resolution terrain model for each attribute.

\section{2. $2 D$ raster surfaces generation}

In Step 2 of our algorithm, we generate 2D elevation, reflectance and pulse width raster surfaces from the first level terrain pyramids using natural neighbourhood interpolation (Crawford, 2009). The first level terrain pyramid is found to be useful for generating the smooth raster surface, as it minimises the noise effect without affecting much of the object details and accuracy. The cell size, $c$ parameter required to generate the raster surfaces is selected empirically.

We generate a slope raster surface from the elevation surface. The slope values are estimated as the rate of change in elevation of the raster cells to its neighbours (ESRI, 2010). A plane is fitted to the $z$ elevation values of a $3 \times 3$ neighbourhood around each raster cell and then its slope value is calculated as the rate of change of $z$-values in the horizontal $x$ and the vertical $y$ direction, which can be described as

$\arctan \left(\sqrt{\left(\frac{d z}{d x}\right)^{2}+\left(\frac{d z}{d y}\right)^{2}}\right)$.

The slope, reflectance and pulse width raster surface values are normalised with respect to their global minimum and maximum values, and converted to an 8-bit data type. This allows for a two-way transformation between the 8-bit values and their original LiDAR values for all road sections. This in turn will allow for the use of a single threshold value for all road sections. Examples of the slope, reflectance and pulse width raster surfaces generated from their respective terrain pyramids are shown in Fig. 2.

\subsection{Snake energy estimation}

A snake curve is defined within a $2 \mathrm{D}$ raster surface domain that moves under the influence of internal and external energy. In Step 3 of our road edge extraction algorithm, we estimate these energy terms before we initialise the snake in Step 4. We make novel use of the balloon and GVF models in our algorithm. We combine both the models such that the balloon external energy pushes the snake curve and the GVF external energy attracts the snake curve toward the object boundaries. The GVF external energy terms are computed as diffused energy field of the gradient vectors of the object boundaries from the raster surfaces (Xu et al., 1997). To compute the GVF energy, the object boundaries are determined from the slope, reflectance and pulse width raster surfaces respectively through the consecutive use of hierarchical thresholding and Canny edge detection.

Hierarchical thresholding segments the input raster surfaces by thresholding a hierarchy of low to high resolution versions of each raster surface with a threshold, $T$ parameter (Sonka et al., 2008). This hierarchy is generated by blurring each input raster surface with a Gaussian filter which convolves each block in the raster surface with a kernel that consists of a matrix of numbers (Fisher et al., 2000). A mask size, $M$, of the kernel describes $M \times M$ matrix of numbers and a block represents $M \times M$ group of cells in the raster surface. The value of each convolved block is obtained by multiplying each kernel element with its underlying raster cell value and then adding them together. An advantage of using the hierarchical thresholding approach is its ability to provide a better estimation of the object in the presence of noise. This makes a single threshold value more robust, which in turn, allows for the hierarchical threshold to be applicable to our automated algorithm. The mask size, $M_{\text {slope }}, M_{r e f}, M_{p w}$ and threshold, $T_{\text {slope }}, T_{r e f}, T_{p w}$ parameters that are applied to the slope, reflectance and pulse width raster surfaces in the hierarchical thresholding are selected empirically. Their values are fixed for all the road sections, which allows for the fully automatic application of hierarchical thresholding. An optimal selection of threshold parameters is important as they impact the final outcome of the algorithm. To demonstrate their importance, we applied a lower, optimal and higher value of $T_{\text {slope }}$ to the slope raster surface as 25,50 and 125 , shown in Fig. 3 . The use of a lower threshold value led to the estimation of object cells in between the possible road edges as shown in Fig. 3(a). These object cells can create an obstruction for the snake curve to move towards the road edges. The use of its higher value removed the possible road edge object cells as shown in Fig. 3(c). It can cause the snake curve to move beyond and falsely identifying the road edges. Thus, the use of optimal threshold value as in Fig. 3(b), is essential for a proper estimation of object cells which impact the road edge extraction results. The hierarchical thresholding approach outputs raster surfaces with only two values, 255 for object cells and 0 for non-object cells. We input these surfaces into the Canny edge detection to determine object boundaries.

In the Canny edge detection approach, each raster surface is blurred by applying a Gaussian filter and then a Sobel operator is applied to determine gradients (Canny, 1986; Moeslund, 2008).

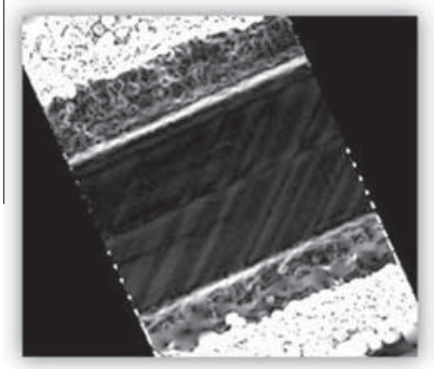

(a)

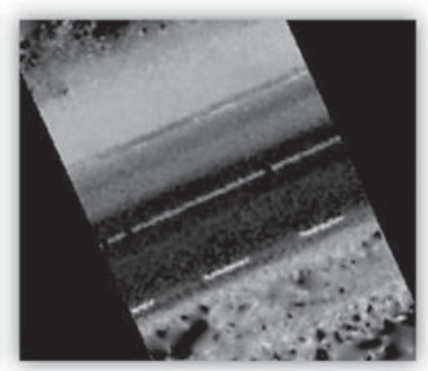

(b)

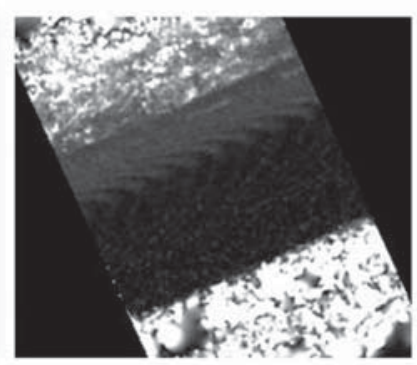

(c)

Fig. 2. 2D raster surfaces generated from their respective terrain pyramids: (a) slope, (b) reflectance and (c) pulse width. 


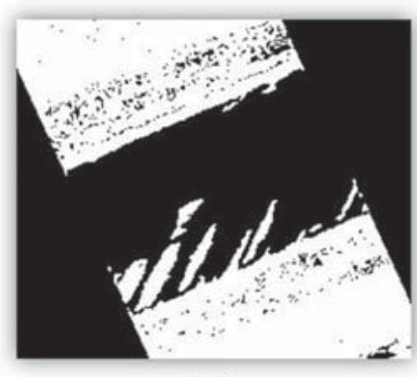

(a)

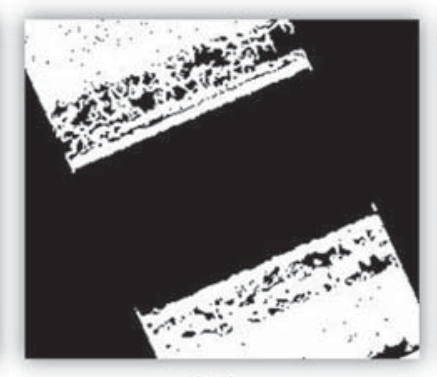

(b)

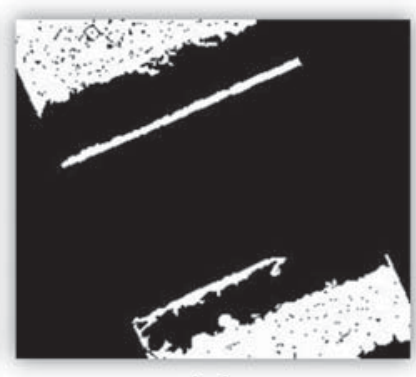

(c)

Fig. 3. Threshold parameter, $T_{\text {slope }}$, applied to the slope raster surface with (a) lower, (b) optimal and (c) higher value.

In the next step, local maximum values of the gradients are estimated by comparing the value of each cell with its neighbours along the gradient direction. The estimated local maximum gradient values are preserved and non-maximum values are removed from each raster surface. After this, upper threshold, $T_{1}$ and lower threshold, $T_{2}$ parameters are applied to each raster surface which provide an estimation of the object boundaries, $f_{\text {slope }}, f_{r e f}$ and $f_{p w}$. We set $T_{1}=250$ and $T_{2}=5$ based on the output cell values obtained from the hierarchical thresholding. This makes our Canny edge detection fully automated and much more robust to noise. An example of the object boundaries, $f_{\text {slope }}$, estimation from the slope raster surface through the consecutive use of hierarchical thresholding and Canny edge detection is shown in Fig. 4.

After determining the object boundaries, $f_{\text {slope }}, f_{\text {ref }}$ and $f_{p w}$, from the slope, reflectance and pulse width raster surfaces, the GVF external energy terms are estimated by iteratively diffusing their gradient vector values. This iterative process is used to estimate the appropriate GVF energy terms required for attracting the snake curve towards the object boundaries. Finally, the balloon energy is included in the external energy by providing a weight to the normal unit vector of the snake curve points that helps push it outwards. Internal energy is provided to the snake curve by adjusting its elasticity and stiffness properties. The snake's energy function $E_{\text {snake }}$ can be described as

$E_{\text {snake }}=E_{\text {int }}+E_{\text {balloon }}+\kappa_{2} \cdot E_{G V F(\text { slope })}+\kappa_{3} \cdot E_{G V F(r e f)}+\kappa_{4} \cdot E_{G V F(p w)}$,

where $E_{\text {balloon }}$ is the balloon energy as mentioned in Eq. (6). $E_{G V F(\text { slope }),} E_{G V F(r e f)}$ and $E_{G V F(p w)}$ are the GVF external energy terms estimated from the slope, reflectance and pulse width raster surfaces respectively in accordance with Eq. (7), while $\kappa_{2}, \kappa_{3}$ and $\kappa_{4}$ are their respective weight parameters. The weight parameters for the balloon and GVF energy terms are selected empirically after examining their several combinations and are fixed for all the road sections.

\subsection{Snake curve initialisation}

In Step 4 of our algorithm, we initialise the snake curve over a 2D raster surface using the navigation track of the mobile van along the road section. We choose a parametric ellipse for initialising the snake curve as shown in Fig. 5.

The snake points, $(x, y)$, are expressed in the form of parametric ellipse as

$$
x(t)=x_{c}+a \cos (\delta \lambda) \cos (\phi)-b \sin (\delta \lambda) \sin (\phi)
$$$$
y(t)=y_{c}+a \cos (\delta \lambda) \sin (\phi)+b \sin (\delta \lambda) \cos (\phi)
$$

where $\delta \lambda$ is the parametric angle interval which is used to provide the $m$ number of points in the snake curve from 0 to $2 \pi$ radian, $\phi$ is the angle between the $X$-axis and major axis of the ellipse and $\left(x_{c}, y_{c}\right)$ is the centre point of the ellipse. The semi-major axis of the snake ellipse, $a$, is computed as the difference between the middle and the first or last navigation points, described in Fig. 5(a). The centre of the road is estimated based on the $\omega$ angle in between the major axis and the line segment from the first or last navigation point, shown in Fig. 5(b). This angle is selected empirically and fixed for the road sections with similar width. The semi-minor axis of the snake ellipse, $b$, is computed as $a \tan \omega$. The $\phi$ angle is calculated from the $\theta$, average heading angle of the mobile van along the road section under investigation, as described in Fig. 6. The average heading angle is estimated as a mean of the mobile van's heading angle along the road section under investigation. If the value of $\theta$ angle lies in between $0^{\circ}$ and $90^{\circ}$ angles or between $90^{\circ}$ and $180^{\circ}$ angles, then the $\phi$ angle is estimated as $90^{\circ}-\theta$, as shown in Fig. 6(a) and (b). Similarly, the $\phi$ angle can be estimated for other possible values of the $\theta$ angle, shown in Fig. 6(c) and (d). Thus, the estimation of $\phi$ angle is completely automated in our algorithm. Considering the $10 \mathrm{~m}$ length of the input road section in the algorithm, the value of average heading would be stable with respect to the individual heading values and would not impact the efficiency of initialisation process.

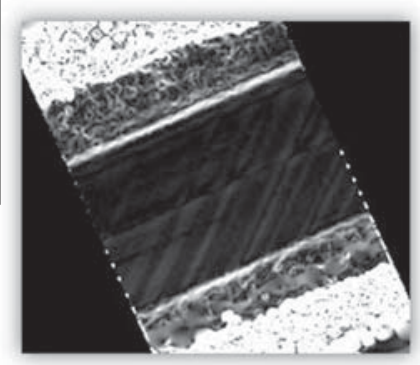

(a)

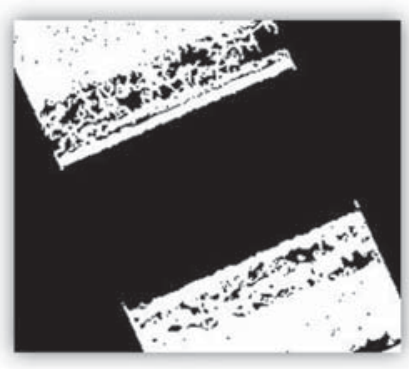

(b)

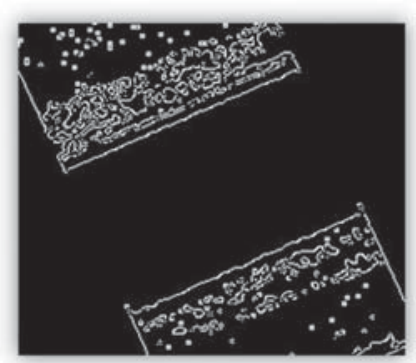

(c)

Fig. 4. Object boundaries, $f_{\text {slope }}$, estimation: (a) slope raster surface, (b) hierarchical thresholded objects and (c) their boundaries. 


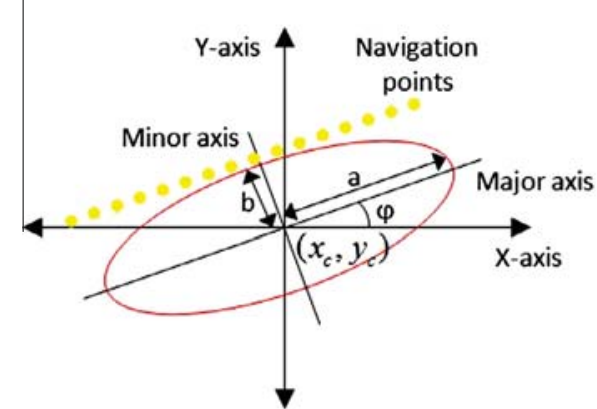

(a)

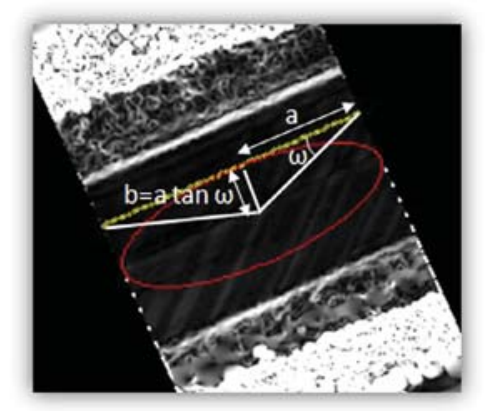

(b)

Fig. 5. Snake curve initialisation in (a) parametric ellipse form and (b) centre of the road estimation.

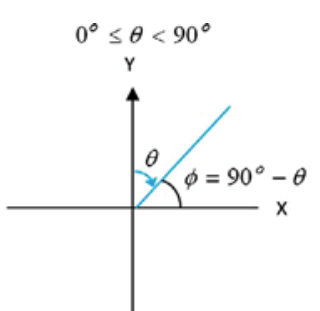

(a)

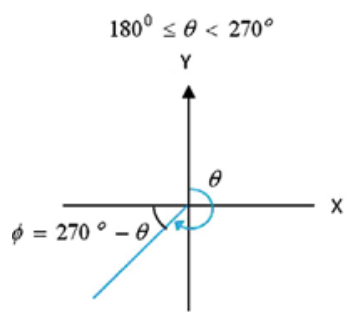

(c)

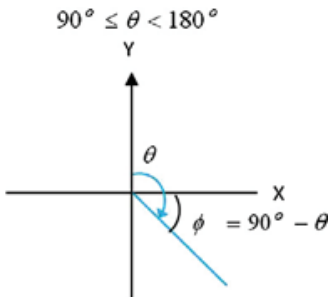

(b)

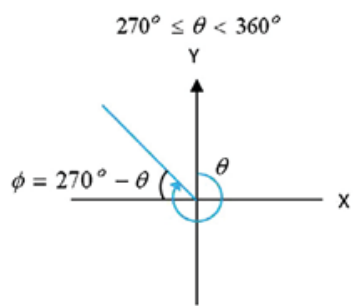

(d)
Fig. 6. $\phi$ Angle is calculated from $\theta$, which is an average heading angle of the mobile van along the road section under investigation that can lie in between (a) $0^{\circ}$ and $90^{\circ}$, (b) $90^{\circ}$ and $180^{\circ}$, (c) $180^{\circ}$ and $270^{\circ}$ and (d) $270^{\circ}$ and $360^{\circ}$.

\subsection{Final snake curve}

In Step 5 of our road edge extraction algorithm, the snake curve moves under the influence of the internal and external energy terms. During an iterative process it approaches the minimum energy state and converges to the road edges. Examples of the initial, iterative and final positions of the snake curve are shown in Fig. 7.

\subsection{Overlapping snake curves}

In Step 6 of our algorithm, after each road section has been processed we extract the left and right road edges. We obtain overlapping snake curves by batch processing consecutive individual road sections as shown in Fig. 8. We then find the intersection points in between the overlapping snake curve points using their parametric line equations. The intersection points with the highest and lowest Northing are then selected, as shown in blue circles in Fig. 8(a) and (b). In Fig. 8(a) and (b), the non-road edge points in between the circled intersection points are removed clockwise in the first snake curve and anti-clockwise in the next snake curve resulting in one continuous snake for the complete road section as shown in Fig. 8(c). This process verifies our selection of consecutive and overlapped road sections as an input in the algorithm.

\section{7. $3 D$ road edges}

In Step 7 of our road edge extraction algorithm, we provide the third dimension to the extracted left and right road edge points. We provide the elevation value from the nearest LiDAR point to the road edge point. In the next section, we describe the approach for validating the extracted road edges.

\section{Validation approach}

We validate our automated road edge extraction results through their comparison with the manually digitised road edges. We create buffer zones around the manually digitised left and right edges, which are considered as ground truth in each road section and then the automated extracted left and right edges are labelled as inside or outside the buffer zone (Heipke et al., 1997; Zhou and Vosselman, 2012). Examples of 0.1 and $0.3 \mathrm{~m}$ buffer width around the digitised edges are shown in Fig. 9. The extracted road edges are evaluated by calculating the quality measures, completeness and correctness. The completeness is calculated as

$\frac{\sum_{i=0}^{k-1} E}{\sum_{i=0}^{r-1} G T}$

where $k$ is the number of extracted edge points inside the buffer zone, $E$ is the length of extracted edge, $r$ is the total number of ground truth or digitised edge points and GT is the length of ground truth edge. In some of our tested road sections, the length of extracted edge inside the buffer zone was more than the length of ground truth edge, which led to an inappropriate completeness measure of more than 1 . This increased length was due to the jagged extraction of edge in those sections in comparison to their manual digitisation. We modified the Eq. (12) as

$\frac{\sum_{i=0}^{k-1} E}{\sum_{i=0}^{p-1} G T+\sum_{i=0}^{q-1} G T}$

where $p$ is the number of ground truth edge points corresponding to $k$, the extracted edge points inside the buffer zone and $q$ is the number of ground truth edge points corresponding to $(s-k)$, the extracted edge points outside the buffer zone with $s$ being the total number of extracted edge points. These ground truth edge points are equated with the extracted edge points on the basis of their location while sidelining the jagged edge points which are unlikely to be in the real world environment. Eq. (13) can be modified as

$\frac{\sum_{i=0}^{k-1} E}{\sum_{i=0}^{k-1} E+\sum_{i=0}^{q-1} G T}$

which is used to calculate the completeness in our validation approach. The correctness is computed as 


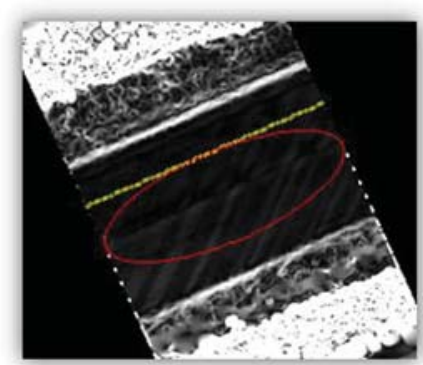

(a)

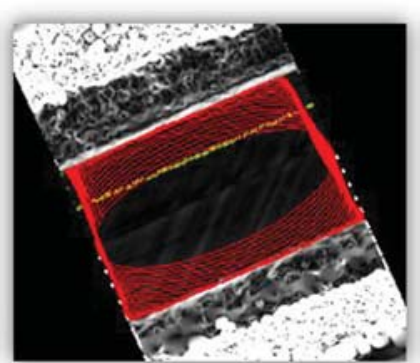

(b)

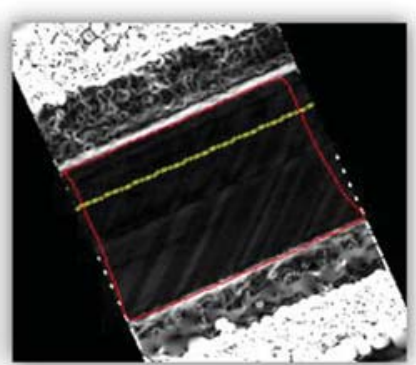

(c)

Fig. 7. Snake curve positions: (a) initial, (b) iterative and (c) final.

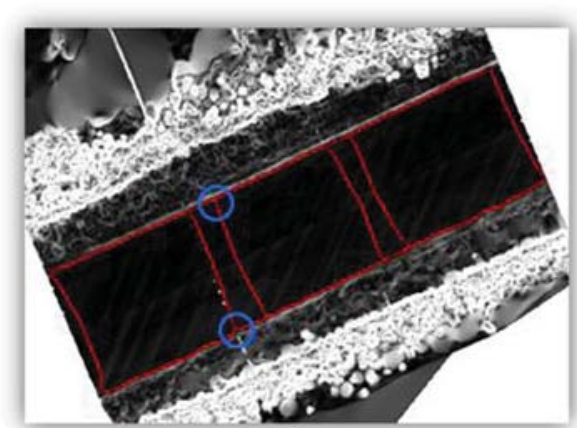

(a)

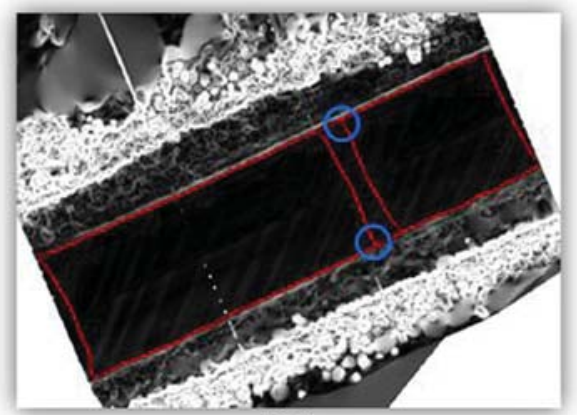

(b)

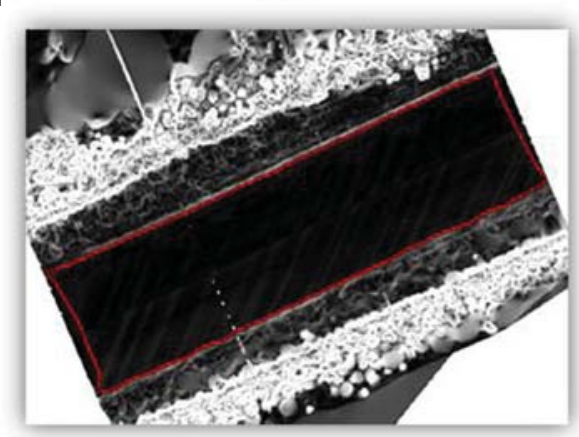

(c)

Fig. 8. Three processed road sections with (a) first intersection points highlighted, (b) second intersection points highlighted and (c) final left and right road edges.

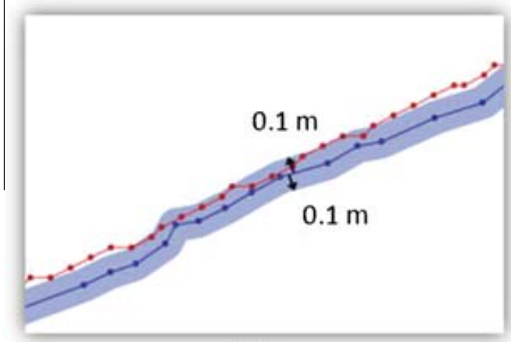

(a)

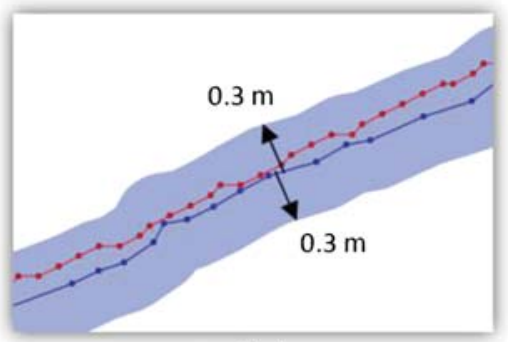

(b)

Fig. 9. The buffer zones of (a) 0.1 and (c) $0.3 \mathrm{~m}$ width created around the manually digitised edges represented with blue, while the automated extracted edges are represented with red. (For interpretation of the references to colour in this figure legend, the reader is referred to the web version of this article.)

$\frac{\sum_{i=0}^{k-1} E}{\sum_{i=0}^{s-1} E}$

In the next section, we present the experiments of our automated algorithm on different types of road sections.

\section{Experimentation}

We selected three sections of road to test our road edge extraction algorithm. These sections covered $150 \mathrm{~m}$ of rural, urban and national primary roads which consisted of three types of road 


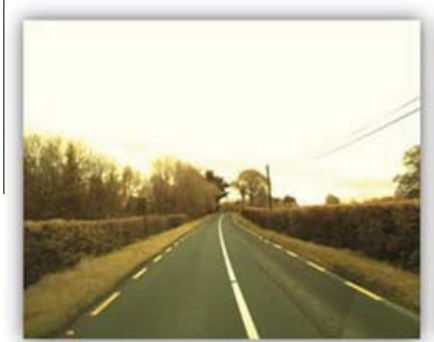

(a)

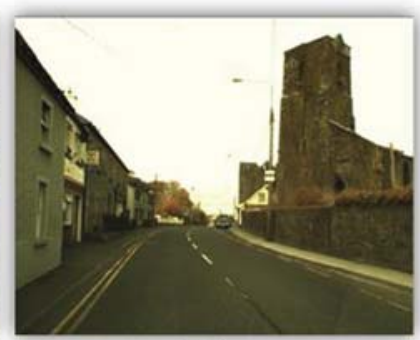

(b)

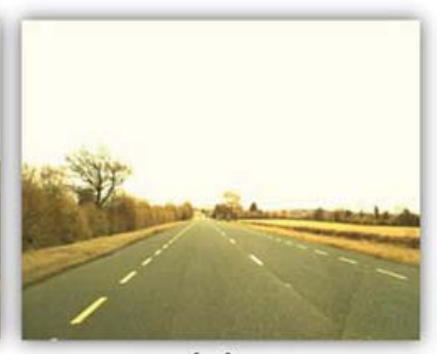

(c)

Fig. 10. Digital image of the (a) rural road section consisting of grass-soil edges (Geographic location: $53^{\circ} 34^{\prime} 28.07^{\prime \prime} \mathrm{N} 7^{\circ} 10^{\prime} 13.76^{\prime \prime} \mathrm{W}$ ), (b) urban road section consisting of kerb edges (Geographic location: $53^{\circ} 36^{\prime} 37.42^{\prime \prime} \mathrm{N} 7^{\circ} 5^{\prime} 41.00^{\prime \prime} \mathrm{W}$ ) and (c) national primary road section consisting of grass-soil edges with shoulders (Geographic location: $\left.53^{\circ} 38^{\prime} 8.80^{\prime \prime} \mathrm{N} 7^{\circ} 29^{\prime} 11.06^{\prime \prime} \mathrm{W}\right)$.

Table 1

The empirically selected parameters used in the road edge extraction algorithm.

\begin{tabular}{llllll}
\hline Parameter & Value & Parameter & Value & Parameter & Value \\
\hline$c$ & $0.06 \mathrm{~m}^{2}$ & $T_{p w}$ & 65 & $\kappa_{1}$ & 1 \\
$M_{\text {slope }}$ & 25 & $T_{1}$ & 250 & $\kappa_{2}$ & 4 \\
$M_{\text {ref }}$ & 25 & $T_{2}$ & 5 & $\kappa_{3}$ & 2 \\
$M_{p w}$ & 25 & $\alpha$ & 9 & $\kappa_{4}$ & 2 \\
$T_{\text {slope }}$ & 50 & $\beta$ & 0.001 & $\delta \lambda$ & 0.03 \\
$T_{\text {ref }}$ & 100 & $\gamma$ & 3 & $\mu$ & 0.2 \\
\hline
\end{tabular}

Table 2

Average heading angle, $\theta$, in each navigation section of the rural, urban and national primary road sections.

\begin{tabular}{llll}
\hline Navigation section & Rural road & Urban road & National primary \\
\hline 1 & $65.59^{\circ}$ & $51.90^{\circ}$ & $127.27^{\circ}$ \\
2 & $65.77^{\circ}$ & $51.59^{\circ}$ & $127.10^{\circ}$ \\
3 & $65.97^{\circ}$ & $51.62^{\circ}$ & $126.90^{\circ}$ \\
4 & $66.24^{\circ}$ & $51.64^{\circ}$ & $126.67^{\circ}$ \\
5 & $66.46^{\circ}$ & $51.34^{\circ}$ & $126.41^{\circ}$ \\
6 & $66.85^{\circ}$ & $50.08^{\circ}$ & $126.16^{\circ}$ \\
\hline
\end{tabular}

edges. We selected these road sections to demonstrate the ability of our algorithm to extract distinct road edges without manual intervention. The processed data was acquired using the eXperimental Platform (XP-1) MMS, which has been designed and developed at NUIM (Kumar et al., 2011).

The first $50 \mathrm{~m}$ section of rural road consisted of grass-soil edges, the second $50 \mathrm{~m}$ section of urban road consisted of kerb edges and the third $50 \mathrm{~m}$ section of national primary road consisted of grass-soil edges with shoulders as shown in Fig. 10. To process each $50 \mathrm{~m}$ road section, we used $n=6$ number of $30 \times 10 \times 5 \mathrm{~m}$ sections of LiDAR data and $n=6$ number of $10 \mathrm{~m}$ section of navigation data.

We applied our road edge extraction algorithm to each road section with the empirically selected parameters as shown in Table 1 . The $\phi$ angle used to initialise the snake curve was calculated as $90^{\circ}-\theta$ in each navigation section of the road sections. The value of $\phi$ angle in each navigation section of the rural, urban and national primary road sections are shown in Table 2.

We did not provided highest weight to the reflectance GVF energy and the pulse width GVF energy. The reflectance values provided by the Riegl VQ-250 laser scanner are not accurately normalised, which leads to different values over the road surface. In most cases, the hierarchical thresholding applied to the reflectance raster surface failed to remove the road marking cells near the road edge points. These road marking cells create an obstruction for the snake curve to move towards the road edges. The orientation of top

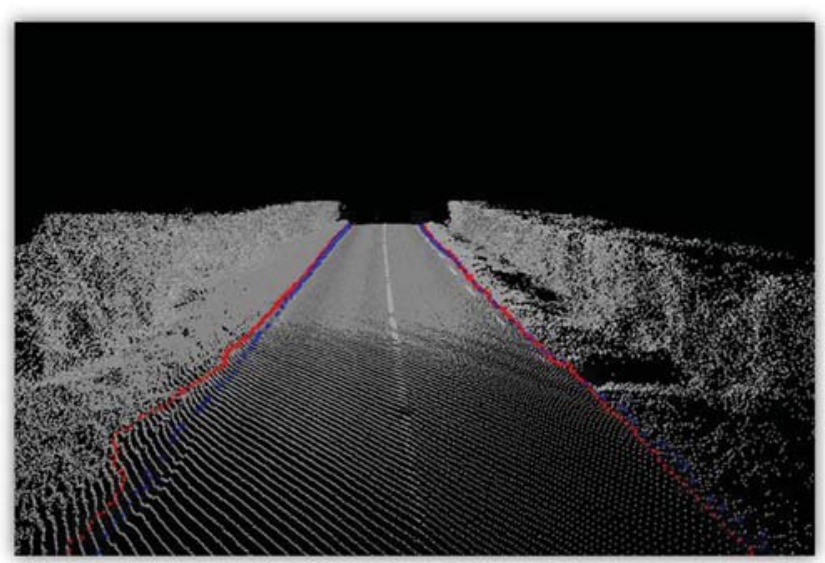

Fig. 11. The automated extracted $3 \mathrm{D}$ edges represented in red and the manually digitised 3D edges represented in blue in the $50 \mathrm{~m}$ rural road section. (For interpretation of the references to colour in this figure legend, the reader is referred to the web version of this article.)

of the kerb edges relative to the vehicle is very similar to that of the road surface. This together with their similar surface composition results in the pulse width having similar values in these cases. This leads to pulse width being less important in urban regions.

In the rural and urban road sections, we used $\omega=20^{\circ}$ while the wider national primary road section required an increase of $\omega$ to $34^{\circ}$. The number of GVF iterations were 600 while the number of iterations used to converge the snake curve to the road edges were 40. We manually digitised the left and right edges from the 3D LiDAR data in the rural road section while in the urban and national primary road sections, we asked different users to digitise the road edges. The reason for choosing different users was to remove any bias in the digitisation process of the road sections, as different users might have different perceptions when digitising 3D LiDAR data. The automated extracted $3 \mathrm{D}$ edges are represented in red while the manually digitised 3D edges are represented in blue in the rural, urban and national primary road sections in Figs. 1113 respectively. In the next section, we validate the experimental results and discuss them.

\section{Results and discussion}

We validated our automated road edge extraction results by computing the completeness and correctness quality measures. The buffer width parameter has considerable effect over the evaluation process of the extracted road edges. To analyse this effect, we created the buffer zones of $0.1,0.2,0.3$ and $0.5 \mathrm{~m}$ width which 


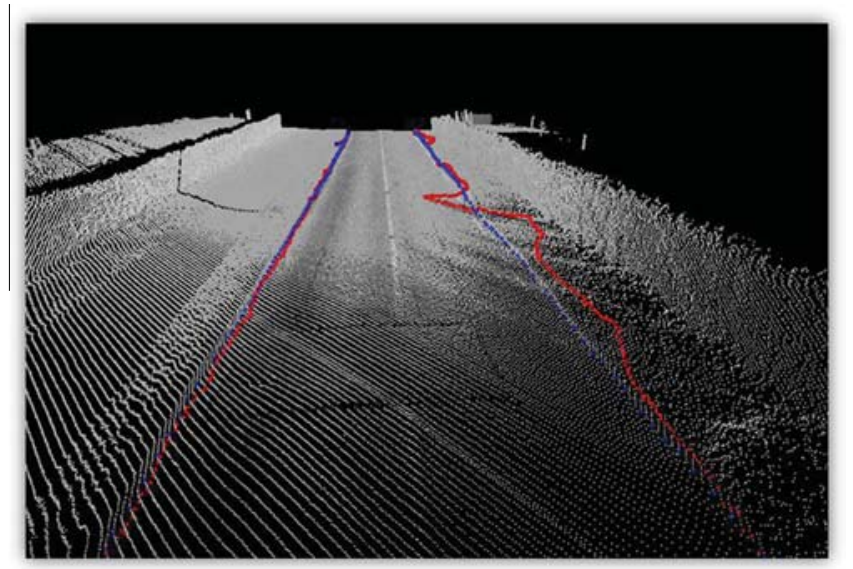

Fig. 12. The automated extracted $3 D$ edges represented in red and the manually digitised 3D edges represented in blue in the $50 \mathrm{~m}$ urban road section. (For interpretation of the references to colour in this figure legend, the reader is referred to the web version of this article.)

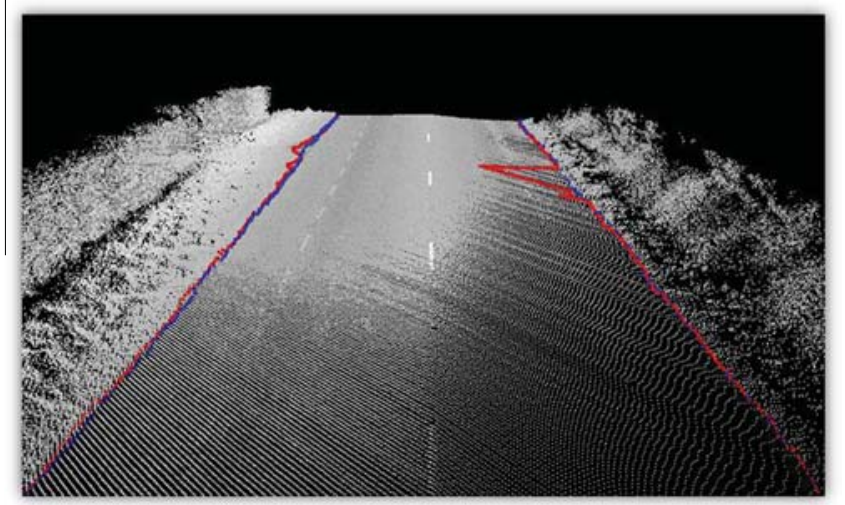

Fig. 13. The automated extracted $3 D$ edges represented in red and the manually digitised 3D edges represented in blue in the $50 \mathrm{~m}$ national primary road section. (For interpretation of the references to colour in this figure legend, the reader is referred to the web version of this article.)

\section{Table 3}

Completeness and correctness values for the automated extracted left and right edges in the rural road section.

\begin{tabular}{llllll}
\hline \multirow{2}{*}{$\begin{array}{l}\text { Buffer width } \\
(\mathrm{m})\end{array}$} & \multicolumn{2}{l}{ Completeness } & & \multicolumn{2}{l}{ Correctness } \\
\cline { 2 - 3 } \cline { 5 - 6 } \cline { 5 - 6 } & $\begin{array}{l}\text { Left edge } \\
(\%)\end{array}$ & $\begin{array}{l}\text { Right edge } \\
(\%)\end{array}$ & & $\begin{array}{l}\text { Left edge } \\
(\%)\end{array}$ & $\begin{array}{l}\text { Right edge } \\
(\%)\end{array}$ \\
\hline 0.1 & 53.4 & 62.5 & & 53.9 & 63.3 \\
0.2 & 78.6 & 97.8 & & 78.8 & 99 \\
0.3 & 90.3 & 98.5 & & 90.6 & 100 \\
0.5 & 96.1 & 98.5 & 96.8 & 100 \\
\hline
\end{tabular}

were selected empirically around the ground truth edges. The completeness and correctness values were calculated for the automated extracted road edges in each road section as shown in Tables 3-5. The quality measure values were found to be higher using the larger buffer width as the extracted edge points farther to the ground truth edge were accepted. The use of smaller buffer width provided lower values of quality measures as nearer extracted edge points were rejected.

Our automated algorithm was able to successfully extract the left and right road edges in all the three tested road sections. In the rural road section, the completeness and correctness values
Table 4

Completeness and correctness values for the automated extracted left and right edges in the urban road section.

\begin{tabular}{llllll}
\hline $\begin{array}{l}\text { Buffer width } \\
(\mathrm{m})\end{array}$ & \multicolumn{2}{l}{ Completeness } & & \multicolumn{2}{l}{ Correctness } \\
\cline { 2 - 3 } \cline { 5 - 6 } & $\begin{array}{l}\text { Left edge } \\
(\%)\end{array}$ & $\begin{array}{l}\text { Right edge } \\
(\%)\end{array}$ & & $\begin{array}{l}\text { Left edge } \\
(\%)\end{array}$ & $\begin{array}{l}\text { Right edge } \\
(\%)\end{array}$ \\
\hline 0.1 & 91.7 & 17.5 & 94.5 & 16 \\
0.2 & 95.6 & 43.8 & & 99.1 & 40.7 \\
0.3 & 96.5 & 53.5 & & 100 & 50.8 \\
0.5 & 96.5 & 65.4 & 100 & 63.8 \\
\hline
\end{tabular}

Table 5

Completeness and correctness values for the automated extracted left and right edges in the national primary road section.

\begin{tabular}{llllll}
\hline $\begin{array}{l}\text { Buffer width } \\
(\mathrm{m})\end{array}$ & \multicolumn{2}{l}{ Completeness } & & \multicolumn{2}{l}{ Correctness } \\
\cline { 2 - 3 } \cline { 5 - 6 } & $\begin{array}{l}\text { Left edge } \\
(\%)\end{array}$ & $\begin{array}{l}\text { Right edge } \\
(\%)\end{array}$ & & $\begin{array}{l}\text { Left edge } \\
(\%)\end{array}$ & $\begin{array}{l}\text { Right edge } \\
(\%)\end{array}$ \\
\hline 0.1 & 67.5 & 70 & 66.9 & 60.7 \\
0.2 & 85.3 & 84.5 & 84.7 & 74.3 \\
0.3 & 87.2 & 86.7 & 87.1 & 76.6 \\
0.5 & 93.1 & 89 & 93.1 & 79.3 \\
\hline
\end{tabular}

for the right edge using $0.2,0.3$ and $0.5 \mathrm{~m}$ buffer width were greater than $97 \%$ as seen in Table 3. The completeness and correctness values for the left edge using 0.3 and $0.5 \mathrm{~m}$ buffer width were greater than $90 \%$ while the values using $0.1 \mathrm{~m}$ buffer width were greater than $53 \%$. The quality measure values obtained for the left edge were lower than for the right edge. The primary reason for this was the incorrect extension of the snake curve into the grass and soil area at some of the points along the left side. This was due to higher elevation difference between the grass and the soil area compared to lower elevation difference between the road surface and the soil. It can be overcome with the use of proper normalised values of intensity in the algorithm which will lead to the generation of a smoother reflectance raster surfaces. The intensity values depend upon the illuminated surface characteristics, the distance from the laser scanner to the illuminated surface and the incidence angle of the laser pulse (Kaasalainen et al., 2011). The intensity values are required to be normalised with respect to these factors. This will allow us to assign a higher weight to the reflectance GVF energy in the algorithm. This will be useful in road sections consisting of grass-soil edges where elevation difference between the road surface and the grass-soil surface is negligible.

In the urban road section, our algorithm produced good results along the left edge as the completeness and correctness values for it were greater than $91 \%$ in all the cases of buffer width shown in Table 4 . The snake curve was also able to identify the drainage system along the left edge as shown in Fig. 14. The right edge extraction results were not accurate as its quality measure values using $0.1 \mathrm{~m}$ buffer width were greater than $16 \%$ while the values using $0.5 \mathrm{~m}$ buffer zone were greater than $63 \%$. This was due to the fact that the XP-1 system employs a single laser scanner during the data acquisition process, which leads to the acquisition of LiDAR data with a lower point density along the right side of the road section than along its left side (Cahalane et al., 2012), as shown for the tested road sections in Table 6. This lower point density along the right side of the road section leads to the generation of raster surfaces with noisy and weak cells along the right road edge. During the object boundaries estimation process in the urban road section, the threshold parameters removed these weak cells along the right road edge, which caused the snake curve to extend beyond them. 


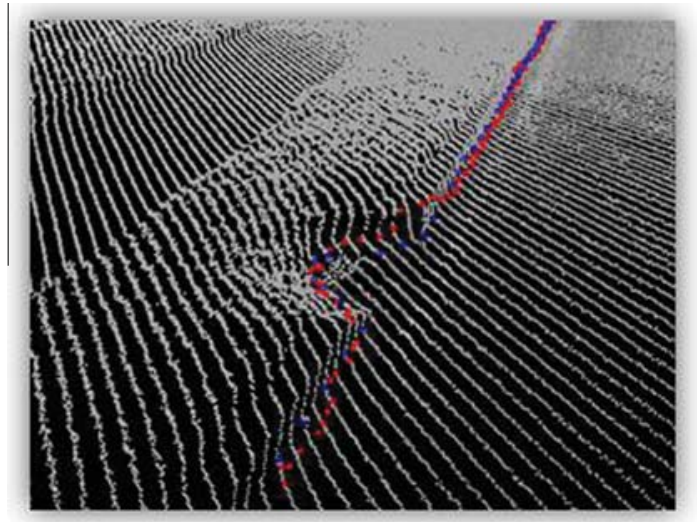

Fig. 14. The drainage system identified by the snake curve along the left edge in the urban road section.

\section{Table 6}

LiDAR point density over the left and right sides of rural, urban and national primary road sections, acquired with the XP-1 system.

\begin{tabular}{lclc}
\hline Road section & $\begin{array}{l}\text { Left side } \\
\left(\text { number of points } / \mathrm{m}^{2}\right)\end{array}$ & $\begin{array}{l}\text { Right side } \\
\left(\text { number of points } / \mathrm{m}^{2}\right)\end{array}$ & $\begin{array}{l}\text { Van speed } \\
(\mathrm{km} / \mathrm{h})\end{array}$ \\
\hline Rural & 702.83 & 127.32 & 61.12 \\
Rural & 697.74 & 137.51 & 60.97 \\
Rural & 702.83 & 138.78 & 61.61 \\
Urban & 861.98 & 143.88 & 51.48 \\
Urban & 884.90 & 140.06 & 51.57 \\
Urban & 895.09 & 142.60 & 51.76 \\
National primary & 1056.79 & 44.56 & 56.66 \\
National primary & 1033.87 & 45.84 & 56.79 \\
National primary & 1004.59 & 47.11 & 56.89 \\
\hline
\end{tabular}

Zhou and Vosselman, 2012 reported an average completeness and correctness values of $63 \%$ and $89 \%$ respectively using $0.5 \mathrm{~m}$ buffer width for the kerbstones extracted from mobile laser scanning data. In comparison, our algorithm extracted the kerb edges with the average completeness and correctness values of $81 \%$ and $82 \%$ respectively using $0.5 \mathrm{~m}$ buffer width. Present day terrestrial mobile laser scanning systems used in professional road surveys are generally equipped with more than one laser scanner. Their solution produces LiDAR data that has a more uniform point density along both sides of the road section. A double pass approach in which the vehicle is drived back and forth on the road can also be used to acquire uniform and dense point cloud along the road section. Our algorithm will provide an improved extraction of road edges using such LiDAR data.

In the national primary road section, the completeness and correctness values for the left edge and the completeness values for the right edge using $0.2,0.3$ and $0.5 \mathrm{~m}$ buffer width were greater than $84 \%$ as seen in Table 5 . The correctness values obtained for the right edge were lower than for the left edge as the snake curve was not able to reach the right edge in one of the road section shown in Fig. 15(a). This was due to lower point density along the right side of this road section which resulted in significant amount of noisy cells in between the road edge points in the slope raster surface generated from the LiDAR elevation attribute. The hierarchical thresholding applied to the slope raster surface was not able to remove the noisy cells. These noisy cells created an obstruction for the snake curve to move towards the right road edges. This noise can be overcome by providing an increased weight to the balloon energy with $\kappa_{1}=4$ parameter. It provided an additional inflation energy to the snake curve to move towards the right road edge as shown in Fig. 15(b). However, the increased balloon energy pushed the snake curve beyond the weak left edge points. An efficient use of the balloon energy weight parameter in the algorithm is required to be explored in order to provide proper inflation energy to the snake curve. In the next section, we conclude our research work presented in this paper.

\section{Conclusions}

We presented our algorithm in Section 4, based on the novel combination of GVF and balloon parametric active contour models, to extract road edges from the terrestrial mobile LiDAR data. Our algorithm was based on the assumption that the LiDAR elevation, reflectance and pulse width attributes can be used to distinguish the road surface from the grass-soil and the kerb edges. The successful extraction of these road edges from the multiple distinct tested road sections validates our automated algorithm. These findings and knowledge provide valuable insights as well as a prototype road edge extraction tool set, for both national road authorities and survey companies.

Through the consecutive use of hierarchical thresholding and Canny edge detection approaches, we made the threshold parameters estimation process more robust, which in turn facilitated integration with our automated road edge extraction algorithm. Our novel initialisation approach negated the limitation associated with the manual intervention generally required in the parametric active contour models. The internal and external energy parameters used in the algorithm were selected empirically, however they

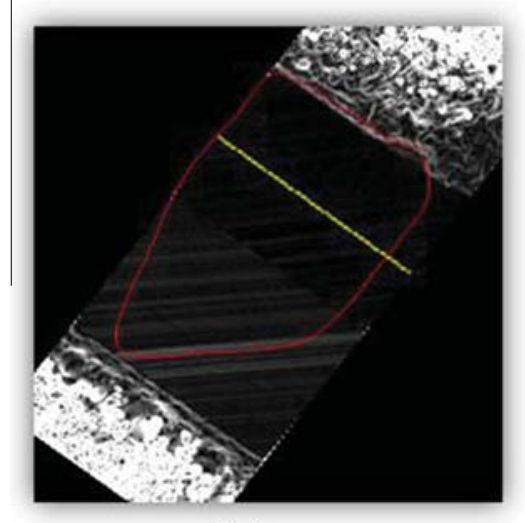

(a)

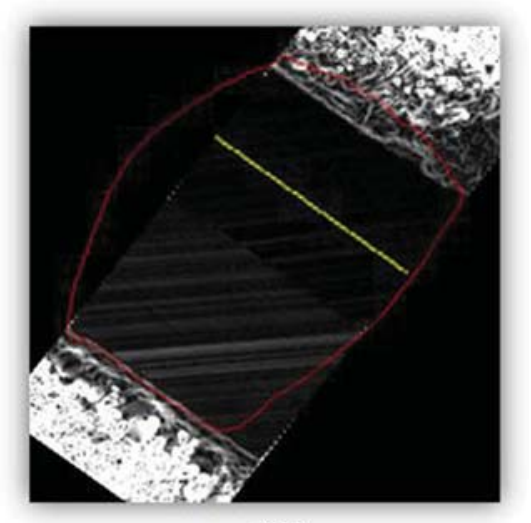

(b)

Fig. 15. Final position of the snake curve over the slope raster surface with (a) $\kappa_{1}=1$ and (b) $\kappa_{1}=4$ parameter in one of the national primary road section. 
are required to be analysed experimentally in order to find their robustness. The road edge extraction results were validated by computing the quality measure values but they provide an aggregate evaluation of the extracted road edge points rather than evaluating individual road edge point. An appropriate selection of buffer width during the validation process is another issue. If the buffer width is large, false extractions will be incorrectly considered as road edges but if its value is small, correct extractions will be rejected. There is a need to develop an efficient and robust approach for qualitative validation of the extracted road edges.

\section{Acknowledgements}

Research presented in this paper was funded by the Irish Research Council (IRC) Enterprise Partnership scheme, Pavement Management Service (PMS) Ltd, Strategic Research Cluster Grant (07/SRC/I1168) of Science Foundation Ireland (SFI) under the National Development Plan and National Road Authority (NRA) research fellowship program. The authors gratefully acknowledge this support.

\section{References}

Barber, D., Mills, J., Smithvoysey, S., 2008. Geometric validation of a ground-based mobile laser scanning system. ISPRS Journal of Photogrammetry and Remote Sensing 63 (1), 128-141.

Blake, A., Isard, M., 1998. Active Contours, first ed. Springer, London.

Boyko, A., Funkhouser, T., 2011. Extracting roads from dense point clouds in large scale urban environment. ISPRS Journal of Photogrammetry and Remote Sensing 66 (6), S2-S12.

Cahalane, C., McElhinney, C.P., McCarthy, T., 2010. Mobile mapping system performance - an analysis of the effect of laser scanner configuration and vehicle velocity on scan profiles. In: Proceedings of the European LiDAR Mapping Forum, Hague, 30 November-1 December, pp. 1-10.

Cahalane, C., McElhinney, C.P., McCarthy, T., 2011. Calculating the effect of dual-axis scanner rotations and surface orientation on scan profiles. In: Proceedings of the 7th International Symposium on Mobile Mapping Technology, Krakow, 13-16 June, pp. 1-6.

Cahalane, C., Mccarthy, T., McElhinney, C.P., 2012. MIMIC: mobile mapping point density calculator. In: Proceedings of the 3rd International Conference on Computing for Geospatial Research and Applications, Washington, 1-3 July, pp. $15: 1-15: 9$.

Canny, J., 1986. A computational approach to edge detection. IEEE Transactions on Pattern Analysis and Machine Intelligence 8 (6), 679-698.

Clode, S., Kootsookos, P., Rottensteiner, F., 2004. The automatic extraction of roads from LiDAR data. International Archives of Photogrammetry, Remote Sensing and Spatial Information Sciences 35 (Part B3), 1-6.

Cohen, L.D., 1991. On active contour models and balloons. CVGIP: Image Understanding 53 (2), 211-218.

Crawford, C., 2009. Minimising Noise from LiDAR for Contouring and Slope Analysis. <http://blogs.esri.com/esri/arcgis/2009/09/02> (accessed 10.01.12).

Darnel, C., 2012. Using LiDAR to solve industry challenges. Geo: Geoconnexion International Magazine 11 (1), 18-19.

Elberink, S.J.O., Vosselman, G., 2009. 3D information extraction from laser point clouds covering complex road junctions. The Photogrammetric Record 24 (125), 23-36 (ISSN 0031868X).

Ellum, C., El-Sheimy, N., 2002. Land-based mobile mapping systems. Photogrammetric Engineering \& Remote Sensing 68 (1), 13-17.

ESRI, 2010. Terrain Pyramids. <http://webhelp.esri.com/ArcGISdesktop/9.3> (accessed 28.12.11).

Fisher, R., Perkins, S., Walker, A., Wolfart, E., 2000. Gaussian Smoothing. <http:// homepages.inf.ed.ac.uk/rbf/HIPR2/gsmooth.htm> (accessed 23.02.12).

Goepfert, J., Rottensteiner, F., 2009. Adaption of roads to ALS data by means of network snakes. International Archives of Photogrammetry, Remote Sensing and Spatial Information Sciences 38 (Part 3/W8), 24-29.

Goulette, F., Nashashibi, F., Abuhadrous, I., Ammoun, S., Laurgeau, C., 2006. An integrated on-board laser range sensing system for on-the way city and road modelling. International Archives of Photogrammetry, Remote Sensing and Spatial Information Sciences 36 (Part 1), 1-6.

Haala, N., Peter, M., Kremer, J., Hunter, G., 2008. Mobile LiDAR mapping for 3D point cloud collection in urban areas - a performance test. International Archives of Photogrammetry, Remote Sensing and Spatial Information Sciences 37 (Part B5), 1119-1124.
Heipke, C., Mayer, H., Wiedemann, C., Jamet, O., 1997. Evaluation of automatic road extraction. International Archives of Photogrammetry, Remote Sensing and Spatial Information Sciences 32 (Part 2/3W3), 151-160.

Ibrahim, S., Lichti, D., 2012. Curb-based street floor extraction from mobile terrestrial LiDAR point cloud. International Archives of Photogrammetry, Remote Sensing and Spatial Information Sciences 39 (Part B5), 193-198.

Jaakkola, A., Hyyppä, J., Hyyppä, H., Kukko, A., 2008. Retrieval algorithms for road surface modelling using laser based mobile mapping. Sensors 8 (9), 5238-5249 (ISSN 1424-8220).

Kaasalainen, S., Jaakkola, A., Kaasalainen, M., Krooks, A., Kukko, A., 2011. Analysis of incidence angle and distance effects on terrestrial laser scanner intensity search for correction methods. Remote Sensing 3 (10), 2207-2221.

Kabolizade, M., Ebadi, H., Ahmadi, S., 2010. An improved snake model for automatic extraction of buildings from urban aerial images and LiDAR data. Computers, Environment and Urban Systems 34 (5), 435-441 (ISSN 01989715).

Kass, M., Witkin, A., Terzopoulos, D., 1988. Snakes: active contour models. International Journal of Computer Vision 1 (4), 321-331.

Kumar, P., 2012. Road Features Extraction Using Terrestrial Mobile Laser Scanning System. National University of Ireland Maynooth (NUIM). <http:// eprints.nuim.ie/view/ethesis/author/Kumar_Pankaj.html> (accessed 15.01.13).

Kumar, P., McCarthy, T., McElhinney, C.P., 2010. Automated road extraction from terrestrial based mobile laser scanning system using the GVF snake model. In: Proceedings of the European LiDAR Mapping Forum, 30 November-1 December, Hague, pp. 1-10.

Kumar, P., McElhinney, C.P., McCarthy, T., 2011. Utilizing terrestrial mobile laser scanning data attributes for road edge extraction using the GVF snake model. In: Proceedings of the 7th International Symposium on Mobile Mapping Technology, 13-16 June, Krakow, pp. 1-6.

Lam, J., Kusevic, K., Mrstik, P., Harrap, R., Greenspan, M., 2010. Urban scene extraction from mobile ground based LiDAR data. In: Proc. 5th International Symposium on 3D Data, Processing, Visualization \& Transmission, 17-20 May, Paris, pp. 1-8.

McElhinney, C.P., Kumar, P., Cahalane, C., McCarthy, T., 2010. Initial results from european road safety inspection (EURSI) mobile mapping project. International Archives of Photogrammetry, Remote Sensing and Spatial Information Sciences 38 (Part 5), 440-445.

Moeslund, T.B., 2008. Image and Video Processing, second ed. Aalborg University, Aalborg.

Petrie, G., 2010. An introduction to the technology Mobile Mapping Systems. Geoinformatics 13 (1) 32-43.

Rieger, P., Studnicka, N., Pfennigbauer, M., Ullrich, A., 2010. Advances in mobile laser scanning data acquisition. In: Proceedings of the FIG Congress Facing Challenges - Building the Capacity 11-16 April, Sydney, pp. 1-15.

Rio, R., 2011. 3D Laser Scanning Systems. <http://www.arcweb.com/marketstudies/Pages/3d-Laser-Scanning.aspx> (accessed 13.04.12).

Rutzinger, M., Elberink, S.J.O., Pu, S., Vosselman, G., 2009. Automatic extraction of vertical walls from mobile and airborne laser scanning data. International Archives of Photogrammetry, Remote Sensing and Spatial Information Sciences 38 (Part W8), 7-11.

Smadja, L., Ninot, J., Gavrilovic, T., 2010. Road extraction and environment interpretation from LiDAR sensors. International Archives of Photogrammetry, Remote Sensing and Spatial Information Sciences 38 (Part 3A), 281-286.

Sonka, M., Hlavac, V., Boyle, R., 2008. Image Processing Analysis and Machine Vision, second ed. Thomson Engineering, New York.

Tao, C.V., Li, J., 2007. Advances in Mobile Mapping Technology. Taylor \& Francis Group, London.

Vosselman, G., 2009. Advanced point cloud processing. In: Proceedings of the Photogrammetric Week, 7-11 September, Stuttgart, pp. 137-146.

Vosselman, G., Liang Z, 2009. Detection of curbstones in airborne laser scanning data. International Archives of Photogrammetry, Remote Sensing and Spatial Information Sciences 38 (Part 3/W8), 111-116.

Xu, C., Prince, J.L., 1998. Snakes, shapes and gradient vector flow. IEEE Transactions on Image Processing 7 (3), 359-369 (ISSN 1057-7149).

Xu, C., Prince, J.L., 1997. Gradient vector flow: a new external force for snakes. In: Proceedings of the Computer Vision Pattern Recognition, 17-19 June, San Juan, pp. 66-71.

Yoon, J., Crane, C.D., 2009. Evaluation of terrain using LADAR data in urban environment for autonomous vehicles and its application in the DARPA urban challenge. In: Proc. ICROS-SICE International Joint Conference, 18-21 August, Fukuoka, pp. 641-646.

Yuan, X., Zhao, C.X., Cai, Y.F., Zhang, H., Chen, D.B., 2008. Road-surface abstraction using ladar sensing. In: Proc. 10th International Conference on Control, Automation, Robotics and Vision, 17-20 December, Hanoi, pp. 1097-1102.

Zhang, W., 2010. LiDAR based road and road-edge detection. In: Proc. Intelligent Vehicles Symposium, 21-24 June, San Diego, pp. 845-848.

Zhou, L., Vosselman, G., 2012. Mapping curbstones in airborne and mobile laser scanning data. International Journal of Applied Earth Observation and Geoinformation 18, 293-304. 